\title{
CHAIN CONDITIONS ON SYMMETRIC ELEMENTS ${ }^{1}$
}

\section{SUSAN MONTGOMERY}

ABSTRACT. Recently Britten has proven an analog of Goldie's theorem for the Jordan ring $S$ of symmetric elements in a ring with involution of characteristic not 2. In this paper we first extend Britten's theorem to the situation where $R$ is an arbitrary ring and the Jordan ring is only an ample subring of the symmetric elements. We apply this result to show that if $S$ has ACC on quadratic ideals, then the (Jordan) nil radical of $S$ is nilpotent.

Recently Britten [1] has proven an analog of Goldie's theorem for the Jordan ring of symmetric elements of a ring with involution. More specifically, he has shown that if $2 R=R$ and the symmetric elements $S$ of $R$ are a prime Jordan ring with either ascending or descending chain condition on quadratic ideals, then $S \simeq S_{1}$, the symmetric elements of a *-prime ring $R_{1}$, and $R_{1}$ has a ring of quotients which is *-simple Artinian.

In this paper, we first extend Britten's theorem to the situation where $R$ is an arbitrary ring and the Jordan ring is only an ample subring of the symmetric el ements. The proof given here is considerably shorter than Britten's; the simplification comes from using an idea of Lanski [5] on direct sums of right ideals.

As an application, we show that if $S$ satisfies the ascending chain condition on quadratic ideals, then the nil radical of $S$ is nilpotent. This question remains open for general Jordan algebras.

Before beginning, we need some definitions. $R$ will always denote an associative ring, and $*$ an involution on $R$ (that is, an antiautomorphism of $R$ of period 2). We let $S_{R}=\left\{x \in R \mid x^{*}=x\right\}$ denote the symmetric elements of $R, T_{R}=\left\{x+x^{*} \mid x \in R\right\}$ the traces, and $N_{R}=\left\{x x^{*} \mid x \in R\right\}$ the norms. An ideal $I$ of $R$ is called a ${ }^{*}$-ideal if $I^{*}=I$; thus, $R$ is *-prime if the product of two nonzero *-ideals is nonzero, and $*_{\text {-simple if there are no }}$

Received by the editors August 13, 1973.

AMS (MOS) subject classifications (1970). Primary 16A28, 16A68; Secondary 16 A46.

Key words and phrases. Involution, symmetric elements, Goldie ring, nil radical.

1 This research was supported in part by NSF Grant \#GP 29119-X. 
proper *-ideals. We say $R$ is semiprime if $R$ contains no nilpotent ideals; it is easy to see that a *-prime ring is semiprime.

In what follows, $J$ will denote a special quadratic Jordan ring; that is, $J$ will be an additive subgroup of $R$ closed under the quadratic operator $x U_{y}=y x y$ and the binary composition $x^{2}$. A Jordan subring $J$ of $S_{R}$ is called ample if it contains all norms and traces and if $x J x^{*} \subseteq J$, for all $x \in$ $R$. An additive subgroup $I \subset J$ is a quadratic ideal of $J$ if $J U_{I} \subset I$.

We abbreviate the ascending (descending) chain condition by ACC (DCC). Recall that $R$ is a right Goldie ring if $R$ has ACC on right annihilators and $R$ has no infinite direct sums of right ideals.

We begin with an easy lemma. Part (ii) is part of Britten's Lemma A [1].

Lemma 1. Let $R$ be a semiprime ring with *, and $J$ an ample Jordan subring of $S$. Then:

(i) If $a \in J$ with $a J a=0$, then $a=0$.

(ii) If $I$ is a right (left) ideal of $R$ with $I \cap J=0$, then $Q=\left\{x+x^{*}\right\}$ $x \in I\}$ is a quadratic ideal of $J$.

Proof. (i) Since $a \in J$ and $J$ is ample, $x a x^{*} \in J$ for all $x \in R$. Thus, $\operatorname{axax}^{*} a=0$. On the other hand, $x+x^{*} \in J$ so $a\left(x+x^{*}\right) a=0$. This gives $a x a=-a x^{*} a$, and so axaxa $=0$, all $x \in R$. But then $(a x)^{3}=0$; that is, $a R$ is a nil right ideal of $R$ of bounded index. By a theorem of Levitzki [3, p. 1], $R$ has a nilpotent ideal (a contradiction), unless $a=0$.

(ii) Say that $I$ is a right ideal, and choose $a \in I$. Then $a J a^{*} \subseteq I \cap J=$ 0 . Thus, if $s \in J, a^{*} s a \in J$ and $a^{*} s a J a^{*} s a=0$. By (i), $a^{*} s a=0$. Since $s$ was arbitrary in $J, a^{*} J a=0$. It now follows that $J U_{a+a^{*}} \subseteq Q$, for all $a \in I$.

The first theorem extends Theorem 1 of Britten.

Theorem 1. Let $R$ be $a^{*}$-prime ring and $J$ ample. If $J$ has no infinite direct sums of quadratic ideals, then $R$ has no infinite direct sums of right or left ideals.

Proof. Assume that the result is false, that is, $J$ has no infinite direct sums of quadratic ideals but, in $R, \mathbb{N}=\left\{T_{i}\right\}$ is an infinite collection of right ideals whose sum is direct.

We claim that we may assume (by replacing $M$ by an appropriate subset) that any finite sum of ideals in $M$ intersects $J$ only in (0). For, let $\prod_{n}=$ $\left\{T_{n}, T_{n+1}, \cdots\right\}$. Now, if $\prod_{=} \Re_{1}$ fails, there exists a finite sum $S_{1}=$ $T_{1}+T_{2}+\cdots+T_{n_{1}-1}$ such that $S_{1} \cap J \neq(0)$. Then try $\mathbb{M}_{n_{1}}=\left\{T_{n_{1}}, \cdots\right\}$. If $M_{n_{1}}$ fails, there must be a finite sum $S_{2}=T_{n_{1}}+\cdots+T_{n_{2}-1}$ with 
$S_{2} \cap J \neq(0)$. Repeat the argument: if $\prod_{n_{k}}$ fails, there is some $S_{k+1} \neq$ $T_{n_{k}}+\cdots+T_{n_{k+1}-1}$ with $S_{k+1} \cap J \neq(0)$. But if $S_{k}$ exists for all $k$, then the set $\left\{S_{k} \cap J\right\}$ is an infinite set of quadratic ideals of $J$ which form a direct sum, a contradiction. Thus, for some $k$, it must be that in $\Re_{n_{k}}$, no finite sum of right ideals intersects $J$ nontrivially. $\mathbb{N}_{n_{k}}$ is the desired subset.

Now for each $T_{i} \in \mathbb{M}$, consider $Q_{i}=\left\{x+x^{*} \mid x \in T_{i}\right\}$. By Lemma $1, Q_{i}$ is a qua dratic ideal of $J$. Since $J$ has no infinite direct sums of quadratic ideals, there is a finite set $\left\{Q_{1}, \cdots, Q_{n}\right\}$ which is dependent: for some $q_{i} \in Q_{i}$, not all zero, $\Sigma q_{i}=0$. Write $q_{i}=x_{i}+x_{i}^{*}$ for $x_{i} \in T_{i}$ and let $a=\Sigma x_{i} \in \Sigma_{i=1}^{n} T_{i}$. Since there is no infinite direct sum in $\left\{Q_{n+1}, \cdots\right\}$, there must be another finite dependent set $\left\{Q_{n+1}, \cdots, Q_{m}\right\}$. Say that $\Sigma p_{j}=0$ for not all $p_{j} \in Q_{j}$ zero. Let $p_{j}=y_{j}+$ $y_{j}^{*}$ and $b=\Sigma y_{j} \in \Sigma_{n+1}^{m} T_{j}$. Then $a+a^{*}=b+b^{*}=0 ;$ thus $a$ and $b$ are skew and are nonzero by independence of the $T_{k}$.

Now let $r \in R$. Then $a r b+b r^{*} a \in\left(\Sigma T_{i}+\Sigma T_{j}\right) \cap J=(0)$, and so $a r b=$ $-b r^{*} a$. But then $a r b \in\left(\Sigma T_{i}\right) \cap\left(\Sigma T_{j}\right)=(0)$ by the directness of $\left\{T_{k}\right\}$. Thus, $a r b=0$, all $r \in R$. But then $(R a R)(R b R)=0$, and $R a R$ and $R b R$ are *-ideals of $R$ since $a$ and $b$ are skew. By the *-primeness of $R, a=0$, or $b=0$, a contradiction. Thus, $R$ cannot have an infinite direct sum of right ideals.

Now if $U$ is a subset of $R$, we write ann $_{r} U=\{x \in R \mid U x=0\}$, the right annibilator of $U$. Similarly, ann $U$ denotes the left annihilator of $U$. If $A_{1} \subset A_{2} \subset \cdots$ is a properly ascending chain of right annihilators in $R$, let $B_{i}=$ ann ${ }_{l} A_{i}$. Then it is easy to verify that the $B_{i}$ are a properly descending chain of left annihilators and that $A_{i}=\operatorname{ann}_{r} B_{i}$.

The next lemma is also part of Britten's Lemma $A$.

Lemma 2. Let $R$ be *-prime. Then if $C$ is the right (left) annihilator of a nonzero ideal of $R, C \cap C^{*}=0$.

Proof. Let $I$ be the ideal of $R$, so that $I C=0$. Then $C I$ is a right ideal of $R$ with $(C I)^{2}=0$, and so $C I=0$ since $R$ is semiprime. Applying *, we have $I^{*} C^{*}=0$ and thus $\left(I+I^{*}\right)\left(C \cap C^{*}\right)=0$. Since $R\left(C \cap C^{*}\right) R$ is a ${ }^{*}$-ideal of $R$, we must have $C \cap C^{*}=0$ since $R$ is *-prime.

We are now able to extend Britten's Theorem 4. The argument is a simplification of his argument.

Theorem 2. Let $R$ be *-prime and $J$ ample. If $J$ has ACC or DCC on quadratic ideals, then $R$ is a Goldie ring.

Proof. If $J$ has ACC or DCC on quadratic ideals, then it is easy to see 
that $J$ has no infinite direct sums of quadratic ideals. Thus, by Theorem 1 , it suffices to show that $R$ satisfies ACC on right annihilators.

We first treat the case when $J$ has ACC on quadratic ideals. If $R$ is not Goldie, let $A_{1} \subset A_{2} \subset \cdots \subset A_{i} \subset \cdots$ be a proper ascending chain of right annihilators in $R$, and let $B_{i}$ be the corresponding descending chain of left annihilators.

First assume that $A_{i} \cap J \neq 0$ for some $i$. Since the $A_{i}$ are ascending, we may as well assume that $A_{i} \cap J \neq 0$ for all $i$. Then $\left\{A_{i} \cap J\right\}$ is an ascending chain of quadratic ideals of $J$, so for some $m, A_{m} \cap J=A_{m+1}$ $\cap J=\cdots$. We claim that this gives a contradiction. For, choose $a \in A_{m} \cap$ $J$ and $y \in A_{m+1}$. Then $y a+a y^{*} \in A_{m+1} \cap J=A_{m} \cap J$, and so $0=$ $B_{m}\left(y a+a y^{*}\right)=B_{m} y a$, since $a \in A_{m}=\operatorname{ann}_{r} B_{m}$. Thus $\left(B_{m} A_{m+1}\right) a=0$. Since $B_{m} A_{m+1} \neq 0$, and $a \in J \subseteq S, a=0$ by Lemma 2. Thus $A_{m} \cap J=0$.

We may therefore assume that $A_{i} \cap J=0$, for all $i_{\text {. }}$ For each $A_{i}$, let $Q_{i}$ be given as in Lemma 1. Since the $Q_{i}$ are ascending, for some $m, Q_{m}=$ $Q_{m+1}=\cdots$. We claim that $A_{m+1}=A_{m} \cdot$ For, if not, choose $a \in A_{m+1}$, $a \notin A_{m}$. Then $a+a^{*} \in Q_{m+1}=Q_{m}$, so $a+a^{*}=a_{m}+a_{m}^{*}$, for some $a_{m} \in A_{m}$. Thus, $a-a_{m}=-\left(a-a_{m}\right)^{*} \in A_{m+i}, \notin A_{m}$, and is skew. So we may assume that $a$ is skew. Now choose any $y \in A_{m+2^{*}}$. Then $a y^{*}+y a^{*}=a y^{*}-y a \epsilon$ $A_{m+2} \cap J=0$, so $a y^{*}=y a$. But then $B_{m+1} a y^{*}=0=B_{m+1} y a$ since $a \in A_{m+1}$. That is, $\left(B_{m+1} A_{m+2}\right) a=0$. As above, this gives $a=0$, a contradiction. Thus, $A_{m+1}=A_{m}$, which contradicts the $A_{i}$ being a proper chain.

The proof for DCC is very similar; if $B_{i} \cap J=0$ for all $i$, get the $Q_{i}$ 's as above. Then we can choose $a$ skew in $B_{m+1}$, not in $B_{m+2}$. If $y \in B_{m}$, we again have $y^{*} a=a y$, so $a\left(B_{m} A_{m+1}\right)=0$ and $a=0$ as before. Thus, we may assume that $B_{i} \cap J \neq 0$, for some $i$. But the $\left\{B_{i} \cap J\right\}$ are a proper descending chain of quadratic ideals, so for some $m, B_{m} \cap J=B_{m+1} \cap J=\cdots$ and we may assume $B_{m} \cap J \neq 0$. But then, choosing $a \in B_{m} \cap J$ and $y \in B_{m}$, we see that $a\left(B_{m} A_{m+i}\right)=0$, so $a=0$.

We are now able to extend Britten's main theorem:

Corollary 1. Let $J$ be an ample subring of $S_{R}$, where $R$ is a ring with *. If $J$ is prime and has ACC or DCC on quadratic ideals, then $J$ is isomorphic to an ample Jordan subring $J_{1}$ of $a^{*}$-prime Goldie ring $R_{1}$.

Proof. Let $P(R)$ denote the prime radical of $R$. Then by the same proof as [2, Lemma 1], $P(R) \cap J \subseteq P(J)$, the prime radical of $J$. But since $J$ is prime, $P(J)=0$; thus, $P(R) \cap J=0$. Let $R_{1}=R / P(R) ; R_{1}$ is semiprime. Now $J_{1}$, the image of $J$ in $R_{1}$, is isomorphic to $J$ since $P(R) \cap J=0$. Also $J_{1}$ 
is ample since $J$ is $\left(R_{1}\right.$ has an induced involution since $\left.P(R) *=P(R)\right)$.

It remains only to show that $R_{1}$ is actually *-prime (since then $R_{1}$

Goldie follows from Theorem 2). Say $A B=0$; where $A, B$ are $\neq 0$ *-ideals of R. Then $A \cap J \neq 0$ (since if $A \cap J=0, a+a^{*}=0=a^{*} a=a^{2}$, for all $a \in A$, and $R$ would contain a nilpotent ideal). Similarly, $B \cap J \neq 0$. But $A \cap J$ and $B \cap J$ are ideals of $J$, and $(A \cap J) U_{B \cap J}=0$, contradicting $J$ being prime.

Since $R_{1}$ is a *-prime Goldie ring, by Goldie's theorem $R_{1}$ has a ring of quotients $A$ which is semisimple Artinian. It is not difficult to see that in fact $A$ is *-simple (see Britten's Theorem 5). We can therefore show that $J$ has a Jordan ring of quotients.

Corollary 2. Let $J, R, J_{1}$, and $R_{1}$ be as in Corollary 1. Then $J$ has a Jordan ring of quotients $Q(J)$. If $A$ is the associative ring of quotients for $R_{1}$, then $Q(J) \simeq Q\left(J_{1}\right)$ is an ample subring of $S_{A}$, and $Q\left(S_{R_{1}}\right)=S_{A}$. When $2 R=R, Q(J)$ is a simple Jordan ring.

Proof. Using Corollary 1 , we may work entirely in $R_{1}$. We claim that every regular el ement of $J$ is regular in $R_{1}$. For let $r \in J$, regular, and say that $r x=0$ for some $x \in R_{1}$. Then $\left(x+x^{*}\right) U_{r}=0$, so $x+x^{*}=0$. Then $x=$ $-x^{*}$, so $x^{2}=-x x^{*} \in J$. Since $x^{2} U_{r}=0, x^{2}=0$. But this says that the right annihilator of $r$ is nil of index 2 , and so $R_{1}$ contains a nilpotent ideal (a contradiction). Thus, $x=0$. Similarly, $x r=0$ implies $x=0$.

We can now apply [7, Theorem 4.5], which states exactly that $Q\left(S_{R}\right)=$ $S_{A}$, and $Q\left(J_{1}\right)$ is ample whenever $R$ has a ring of quotients $A$ and every element of $J$ is regular in $R$.

The fact that when $2 R=R, Q(J) \simeq S_{A}$ is simple follows from a theorem of Herstein [3, Theorem 2.6].

Having extended Britten's results to arbitrary characteristic, we now apply them to study the nil radical of $S$. Let $N(J)$ denote the nil radical of the Jordan ring $J$. As in Corollary $1, P(R)$ will be the (associative) prime radical of $R$, and $P(J)$ the (Jordan) prime radical of $J$. We work with $J$, rather than $S$, since the symmetric elements might not be preserved under homomorphic images.

Lemma 3. Let $R$ be $a^{*}$-prime ring in which $J$ has either ACC or DCC on quadratic ideals. Then $N(J)=0$.

Proof. Since $J$ is ample and $N(J)$ is an ideal of $J, N(J)$ is a core ideal 
of $S$ (for details see [6, p. 387]). Thus, if $N(J) \neq 0$, there exists a nonzero associative *-ideal $B$ of $R$ such that the core $K_{0}(B) \subseteq N(J)$, where $K_{0}(B)=$ $\left\{b+b^{*}+\Sigma b_{i} b_{i}^{*} \mid b, b_{i} \in B\right\}[6$, Corollary, $\mathrm{p} 387]$. We claim that $B$ must contain a nonzero nil right ideal. If $K_{0}(B)=0$, this is trivial; for $b+b^{*}=$ 0 , or $b^{*}=-b$, for all $b \in B$. But then also $b b^{*}=-b^{2}=0$, and so $B$ itself is nil. We may therefore assume that $K_{0}(B) \neq 0$. Choose $x \in K_{0}(B), x \neq 0$. Then $x^{n}=0, x^{n-1} \neq 0$ for some $n$, so letting $y=x^{n-1}$ we have $y^{2}=0$, $y \neq 0$. Now for any $b \in B, y b+b^{*} y \in K_{0}(B) \subseteq N(J)$, and so for some $k$, $\left(y b+b^{*} y\right)^{k}=0$. Multiplying on the right by $y b$, we see $(y b)^{k+1}=0$; that is, $y B$ is nil.

By Theorem 2, $R$ is a Goldie ring. By Lanski's theorem [4], any nil subring of $R$ is nilpotent. Thus, $B$ contains a nilpotent ideal. But $B$ is an ideal in a semiprime ring, so is itself a semiprime ring, a contradiction. Thus, $N(J)=0$.

Theorem 3. Let $R$ be an associative ring with * such that $S$ has ACC on quadratic ideals. Then the nil radical of $S$ is nilpotent. If $S$ has DCC on quadratic ideals, then the nil radical of $S$ equals the prime radical.

Proof. We will first show that $N(S)=P(S)$ in either situation. We do this by showing that if $P$ is any proper prime ideal of $R$, then $P \supseteq N(S)$. For, consider the ring $\bar{R}=R / P \cap P^{*}$ (possibly $P \cap P^{*}=P=P^{*}$ ); $\bar{R}$ is *prime. Let $J=\bar{S}$, the image of $S$ in $\bar{R}$. Then $J$ is ample, has ACC or DCC on quadratic ideals, and so $N(J)=0$ by the lemma. But $\overline{N(S)} \subseteq N(J)$; thus, $N(S) \subseteq P \cap P^{*} \subseteq P$.

Now $P(R)$ is the intersection of all prime ideals of $R$, and so $N(S) \subseteq$ $P(R)$. By [2, Theorem 3], $P(R) \cap S=P(S)$. But $N(S) \subseteq P(R) \cap S$ and $N(S) \supseteq$ $P(S)$. Thus, $N(S)=P(S)$.

In the case when $S$ has ACC, $P(S)$ will simply be the maximal nilpotent ideal, so $N(S)$ is nilpotent.

\section{REFERENCES}

1. Daniel Britten, On prime Jordan rings $H(R)$ with chain condition, J. Algebra 27 (1973), 414-421.

2. T. S. Erickson and S. Montgomery, The prime radical in special Jordan rings, Trans. Amer. Math. Soc. 156 (1971), 155-164. MR 43 \#306.

3. I. N. Herstein, Topics in ring theory, Univ. of Chicago Press, Chicago, Ill., 1969. MR $42 \# 6018$.

4. C. Lanski, Nil subrings of Goldie rings are nilpotent, Canad. J. Math. 21 (1969), 904-907. MR 40 \# 1428. 
5. C. Lanski, Chain conditions in rings with involution (to appear).

6. Kevin McCrimmon, On Herstein's theorems relating Jordan and associative algebras, J. Algebra 13 (1969), 382-392. MR 40 \#2721.

7. Susan Montgomery, Rings of quotients for a class of special Jordan rings, J. Algebra 31 (1974), 154-165.

DEPARTMENT OF MATHEMATICS, UNIVERSITY OF SOUTHERN CALIFORNIA, LOS ANGELES, CALIFORNIA 90007 